\title{
Deucravacitinib in Moderate to Severe Psoriasis: Clinical and Quality-of-Life Outcomes in a Phase 2 Trial
}

Diamant Thaçi $\cdot$ Bruce Strober $\cdot$ Kenneth B. Gordon · Peter Foley $\cdot$ Melinda Gooderham $\cdot$ Akimichi Morita Kim A. Papp · Lluís Puig · M. Alan Menter · Matthew J. Colombo · Yedid Elbez $\cdot$ Renata M. Kisa · June Ye Andrew A. Napoli $\cdot$ Lan Wei $\cdot$ Subhashis Banerjee $\cdot$ Joseph F. Merola $\cdot$ Alice B. Gottlieb

Received: October 4, 2021 / Accepted: November 16, 2021 / Published online: January 13, 2022

(c) The Author(s) 2022

\section{ABSTRACT}

Introduction: Deucravacitinib is an oral, selective tyrosine kinase 2 inhibitor that demonstrated therapeutic benefit in a Phase 2 clinical trial of adults with moderate to severe plaque

Supplementary Information The online version contains supplementary material available at https:// doi.org/10.1007/s13555-021-00649-y.

D. Thaçi $(\bowtie)$

Institute and Comprehensive Center for Inflammation Medicine, University of Luebeck, Ratzeburger Allee 160, 23538 Luebeck, Germany e-mail: diamant.thaci@uksh.de

B. Strober

Yale University, Central Connecticut Dermatology Research, New Haven, CT, USA

K. B. Gordon

Medical College of Wisconsin, Milwaukee, WI, USA

P. Foley

St Vincent's Hospital Melbourne, Probity Medical Research, Skin Health Institute, The University of Melbourne, Melbourne, VIC, Australia

M. Gooderham

SKiN Centre for Dermatology, Queen's University and Probity Medical Research, Peterborough, ON, Canada

\section{A. Morita}

Nagoya City University, Graduate School of Medical

Sciences, Nagoya, Aichi, Japan psoriasis. This analysis was designed to evaluate the effect of deucravacitinib on additional clinical and quality-of-life (QoL) outcomes and assess the relationship between these outcomes in adults with psoriasis.

Methods: Post-hoc analysis of a 12-week Phase 2 trial was conducted for the three most efficacious dosage groups ( $3 \mathrm{mg}$ twice daily, $6 \mathrm{mg}$ twice daily, $12 \mathrm{mg}$ once daily) and placebo.

K. A. Papp

Clinical Research and Probity Medical Research, Waterloo, ON, Canada

L. Puig

Hospital de la Santa Creu i Sant Pau, Universitat Autònoma de Barcelona, Barcelona, Spain

\section{A. Menter}

Baylor University Medical Center, Dallas, TX, USA

M. J. Colombo · Y. Elbez · R. M. Kisa · J. Ye ·

A. A. Napoli · L. Wei · S. Banerjee

Bristol Myers Squibb, Princeton, NJ, USA

J. F. Merola

Harvard Medical School, Brigham and Women's Hospital, Boston, MA, USA

\section{A. B. Gottlieb}

Icahn School of Medicine at Mount Sinai, New York, NY, USA 
Investigator assessments for efficacy included Psoriasis Area and Severity Index (PASI), body surface area (BSA) involvement, and static Physician's Global Assessment; QoL was assessed using the Dermatology Life Quality Index (DLQI). Treatment responses and their associations were evaluated over time.

Results: Deucravacitinib elicited improvement versus placebo as early as Week 4 for most efficacy measures (including changes in absolute PASI and BSA), with efficacy trends observed from Week 2 to Week 12. Improvements in QoL, assessed by achievement of a DLQI overall score of $0 / 1$ (no effect at all on patient's life), followed a pattern similar to deucravacitinibrelated clinical outcomes over 12 weeks. Overall, patients with greater improvements in psoriasis-related clinical signs and symptoms also reported greater improvement in QoL. However, complete skin clearance was not required for achieving DLQI 0/1.

Conclusion: Deucravacitinib treatment produced early response and similar trends in improvements across multiple efficacy assessments and QoL in moderate to severe plaque psoriasis. Deucravacitinib has the potential to become a promising new oral therapy for this condition.

Trial Registration: ClinicalTrials.gov identifier; NCT02931838.

\section{PLAIN LANGUAGE SUMMARY}

Psoriasis is a skin disease that affects up to $2 \%$ of the population. In psoriasis, red, scaly lesions develop on the skin driven by an aberrant immune response. Psoriasis impacts not only physical and mental health but also quality of life (QoL). Deucravacitinib is being investigated as a treatment for psoriasis. We performed a Phase 2 dose-ranging, placebo-controlled, 12 -week study of deucravacitinib in adults with moderate to severe psoriasis. Patients in the USA, Australia, Canada, Germany, Japan, Latvia, Mexico, and Poland participated. The study showed that oral treatment with deucravacitinib was effective using a disease severity score (percentage of patients with $\geq 75 \%$ reduction from baseline in Psoriasis Area and Severity Index score) at Week 12-placebo 7\% and deucravacitinib $67 \%-75 \%$ for the three highest dosages-and was generally well tolerated. We further analyzed the association between efficacy and a QoL measure, the Dermatology Life Quality Index (DLQI), in patients who received placebo or the most effective dosages of deucravacitinib ( $\geq 3 \mathrm{mg}$ twice daily). Deucravacitinib was effective at the three dosage levels tested. Skin improvement occurred early during treatment and was mirrored by improvements in DLQI score during the 12 weeks of treatment. Although some patients did not have complete clearance of their psoriasis, a large percentage of those patients still achieved considerable improvement in QoL as measured by achieving a DLQI score of 0/1 (i.e., no effect at all on the patient's QoL).

Keywords: Body surface area; Humans; Psoriasis; Quality of life; TYK2 


\section{Key Summary Points}

\section{Why carry out this study?}

Moderate to severe psoriasis is a chronic, systemic, immune-mediated disease that is known to have a substantial impact on patients' physical as well as mental health and quality of life (QoL)

Previous results from this Phase 2 trial in patients with moderate to severe plaque psoriasis demonstrated that treatment with the three highest dosages of the selective tyrosine kinase 2 inhibitor deucravacitinib led to significant improvements in clinical signs and symptoms as well as improvements in QoL measures at 12 weeks compared with placebo

This post hoc analysis from the Phase 2 trial explored temporal response and the relationship between patient-reported outcomes and investigator-assessed clinical measures

\section{What was learned from this study?}

This analysis provided additional evidence that deucravacitinib treatment is associated with early improvements in clinical outcomes from baseline through Week 12, as assessed by improvements from baseline in absolute Psoriasis Area and Severity Index, static Physician's Global Assessment, body surface area involvement, and QoL as assessed by Dermatology Life Quality Index

Improvement in QoL followed a pattern of response to treatment similar to that of the clinical outcomes assessed in this analysis

\section{INTRODUCTION}

Several metrics are utilized to assess the severity of psoriasis, make appropriate treatment decisions, and measure treatment response [1]. The Psoriasis Area and Severity Index (PASI) is a measure of clinical disease severity that is commonly used in clinical trials [2-4]. At least $75 \%$ reduction from baseline in PASI (PASI 75) is considered a clinically relevant treatment response and was the primary endpoint in a Phase 2 dose-ranging, placebo-controlled, 12 -week study of deucravacitinib in adults with moderate to severe psoriasis $[3,5,6]$. Static Physician's Global Assessment (sPGA) is often used to assess disease severity at a specific time point regardless of baseline disease severity $[1,7]$. Another efficacy measure is body surface area (BSA) involvement, which is regarded as a more practical instrument for assessing treatment targets in psoriasis by some dermatologists and other experts [6]. The Dermatology Life Quality Index (DLQI) questionnaire, a patient-reported measurement of health-related quality of life (QoL), is often used in clinical trials to assess measures of bothersome symptoms, such as itch, stinging, and pain, as well as its impact on daily functioning $[8,9]$.

Deucravacitinib is an oral selective tyrosine kinase 2 (TYK2) inhibitor in development for treatment of moderate to severe plaque psoriasis, psoriatic arthritis, ulcerative colitis, Crohn's disease, and lupus (NCT03943147) [10]. The mechanism of action of deucravacitinib is distinct from that of other TYK2 or Janus kinase inhibitors in that deucravacitinib selectively binds to a unique pocket in the regulatory domain of TYK2 instead of the highly conserved active site of the catalytic domain [11], allowing high selectivity of action on TYK2 via allosteric inhibition versus related kinases. This leads to targeted inhibition of immune pathways that are central to psoriasis while limiting off-target effects [11]. In the previously published Phase 2 trial in moderate to severe plaque psoriasis, deucravacitinib treatment resulted in $67-75 \%$ of patients reaching PASI 75 at 12 weeks with the three highest dosages of $3 \mathrm{mg}$ twice daily (BID), $6 \mathrm{mg}$ BID, and $12 \mathrm{mg}$ once daily (QD) 
versus $7 \%$ of patients receiving placebo. The two lowest dosage groups ( $3 \mathrm{mg}$ every other day and $3 \mathrm{mg}$ QD) provided limited efficacy compared with placebo [5]. Deucravacitinib treatment also led to improvements versus placebo at 12 weeks in QoL, as assessed by a DLQI score of 0 or $1(0 / 1)$ [5], and was well tolerated overall. Adverse events were reported in $51 \%$ of patients in the placebo group and in 55\% to $80 \%$ of patients in the deucravacitinib groups, with the highest percentage of adverse events occurring in the group receiving deucravacitinib $6 \mathrm{mg}$ BID. Nasopharyngitis, headache, diarrhea, nausea, and upper respiratory tract infection were the most common adverse events. Serious adverse events and adverse events leading to treatment discontinuation were low and balanced across treatment groups. Deucravacitinibtreated patients (at any dose) did not develop laboratory abnormalities characteristic of Janus kinase inhibitors such as neutropenia, liver enzyme or serum creatinine elevations, or dyslipidemia [5].

This analysis of the Phase 2 trial was conducted to further evaluate the efficacy of deucravacitinib at doses $\geq 3 \mathrm{mg}$ BID using a broad range of clinical assessment tools and to further assess the impact on a patient-reported QoL measure (DLQI) for up to 12 weeks in patients with moderate to severe plaque psoriasis.

\section{METHODS}

\section{Study Design}

This was a post hoc analysis of a randomized, double-blind, placebo-controlled, dose-ranging Phase 2 trial of deucravacitinib in adults with moderate to severe plaque psoriasis (NCT02931838) [5]. The study design has been described previously [5]. Adults were eligible for trial participation if they had moderate to severe plaque psoriasis, as defined by affected BSA $\geq 10 \%$, PASI $\geq 12$, and sPGA score $\geq 3$, and were candidates for phototherapy or systemic therapy. Patients were randomized equally to receive one of five dosages of deucravacitinib (3 mg every other day, $3 \mathrm{mg}$ QD,
$3 \mathrm{mg}$ BID, $6 \mathrm{mg}$ BID, or $12 \mathrm{mg}$ QD) or matching oral placebo for 12 weeks.

\section{Study Ethics}

The trial was conducted in accordance with Good Clinical Practice guidelines defined by the International Council for Harmonisation, the Declaration of Helsinki, and the laws and regulatory requirements of the countries with participating study centers [5]. The study protocol, consent form, and all other documents given to patients were approved by an institutional review board or independent ethics committee at each site (see Supporting Information). Written informed patient consent was obtained prior to study participation.

\section{Assessments}

The assessment tools PASI, sPGA, BSA, and DLQI used in the trial have been previously described [5]. The primary efficacy endpoint for the Phase 2 study was PASI 75 response at Week 12.

Mean percentage changes from baseline in absolute PASI score and in BSA were determined over time through Week 12 . PASI $75, \geq 90 \%$ reduction from baseline in PASI (PASI 90), and $100 \%$ reduction from baseline in PASI (PASI 100) responses and sPGA score of 0 (clear) or 1 (minimal disease) were previously published [5]. Additional post hoc analyses were the percentages of patients who achieved absolute PASI $\leq 1$, absolute PASI $\leq 3$, absolute PASI $\leq 5$, $\mathrm{BSA} \leq 1 \%, \quad \mathrm{BSA} \leq 3 \%$, and $\geq 75 \%$ improvement in the product of sPGA and BSA (sPGA $\times$ BSA 75). The sPGA $\times$ BSA measurement is a validated composite instrument, which is the product of sPGA (range, 0 [clear] to 5 [severe]) and is based on the average of the severity of induration, erythema, and scaling over all psoriatic plaques at a given time point) and BSA [12-15]. The DLQI overall score (range, 0 [no impairment of QoL] to 30 [maximum impairment of QoL]) was analyzed over time. The percentages of patients achieving DLQI 0/1 were compared across categories of absolute 
PASI values and changes from baseline and with BSA involvement.

\section{Statistical Analysis}

In analyses of the Phase 2 trial, mean changes in PASI score, as well as PASI 75 and PASI 90 responses, were similar in the highest dosage groups ( $3 \mathrm{mg}$ BID [ $n=45], 6 \mathrm{mg}$ BID [ $n=45]$, $12 \mathrm{mg}$ QD $[n=44])$; efficacy was limited in the two lowest deucravacitinib dosage groups (3 $\mathrm{mg}$ every other day and $3 \mathrm{mg}$ QD) compared with placebo [5]. These findings provided the rationale for combining data from the three highest dosage groups in subsequent analyses $[5,16]$. The placebo group $(n=45)$ was also included in the analyses for comparative purposes.

Improvement from baseline is presented as percentage change from baseline for absolute PASI and BSA involvement through Week 12. Other outcomes (PASI 90, PASI 100, absolute PASI $\leq 1$, absolute PASI $\leq 3$, absolute PASI $\leq 5$, sPGA, BSA, SPGA $\times$ BSA) at Week 12 are expressed as percentages of patients. All missing values were imputed as nonresponse.

\section{RESULTS}

A total of 267 patients were randomized and treated, and 224 (84\%) completed the 12-week treatment period [5]. The treatment groups were comparable in baseline patient demographics and disease characteristics, as previously reported for absolute PASI score, DLQI score, and BSA involvement [5].

Deucravacitinib treatment groups had a greater mean percentage change from baseline in absolute PASI score than the placebo group at each time point through Week 12, with changes evident as early as the first visit at Week 1; decreases were similar among the three dosage groups (Fig. 1). Mean changes from baseline in BSA percentage were greater in the deucravacitinib groups versus the placebo group at Week $12,-18.6 \quad(75.9 \%$ decrease from baseline), $-17.2 \quad(69.4 \%$ decrease), and -15.2 (73.7\% decrease) in the $3 \mathrm{mg}$ BID, $6 \mathrm{mg}$ BID, and $12 \mathrm{mg}$ QD treatment groups, respectively, compared with -7.7 in the placebo group;
Fig. 2). As with the absolute PASI changes, improvement was seen as early as Week 2 , with similar improvement among the three deucravacitinib dosage groups (Fig. 2).

Responses at Week 12 in patients receiving deucravacitinib compared with placebo for the assessments of PASI 90, PASI 100, absolute PASI $\leq 1$, absolute PASI $\leq 3$, absolute PASI $\leq 5$, and sPGA 0/1 are shown in Fig. 3a-f. The proportions of patients who achieved BSA $\leq 1 \%$ (26.7\% [3 mg BID], 37.8\% [6 mg BID], 38.6\% [12 mg QD]) and BSA $\leq 3 \%$ (51.1\%, 44.4\%, and $56.8 \%$, respectively) were higher in the deucravacitinib groups than in the placebo group $(0 \%$ for $\mathrm{BSA} \leq 1 \%$ and $2.2 \%$ for $\mathrm{BSA} \leq 3 \%$; Fig. 3g). The deucravacitinib dosage groups had a higher percentage of patients who achieved sPGA $\times$ BSA $75 \quad(\geq 75 \%$ improvement from baseline), which has previously been used as an assessment of psoriasis severity and response to therapy $[12,15]$ versus the placebo group (Fig. 3h).

Treatment with deucravacitinib was associated with greater improvement from baseline in QoL versus placebo at each time point, as assessed by the percentage of patients achieving a DLQI overall score of $0 / 1$, which reflects no impact at all on the patient's life [17] (Fig. 4). The percentage of patients achieving a DLQI 0/1 increased by Week 4 for each of the three deucravacitinib dosage groups versus placebo and continued to increase through Week 12 in the two highest dosage groups; the percentage of patients achieving a DLQI $0 / 1$ in the three deucravacitinib dosage groups combined was $17.9 \%$ at Week $4,41.8 \%$ at Week 8 , and $55.2 \%$ at Week 12 versus the placebo group, which was $6.7 \%, 4.4 \%$, and $4.4 \%$, respectively (Fig. 4 ).

Improvements in QoL over 12 weeks followed a similar pattern to the improvements observed for clinical outcomes (PASI 75, PASI 90, and sPGA score of 0 or 1 [0/1]; Fig. 5). The DLQI $0 / 1$ responses were concurrent with increases in the percentages of patients from all three deucravacitinib dosage groups combined achieving PASI $75(0 \%$ at Week $1,70.1 \%$ at Week 12), PASI $90(0 \%$ at Week $1,44.0 \%$ at Week 12), and sPGA $0 / 1$ (0\% at Week $0,71.6 \%$ at Week 12). 
Agreement between QoL and PASI improvements were also observed when the achievement of DLQI 0/1 was stratified by absolute PASI bands $(\leq 1 ; \leq 2 ; \leq 3 ; \leq 5)$ at Week 12 , suggesting that the percentage of patients achieving DLQI 0/1 was highest in those with absolute PASI $\leq 1$ (Fig. 6a) and PASI 100 (Fig. 6b). Similarly, higher rates of achievement of DLQI $0 / 1$ were observed in the BSA $\leq 1 \%$ group $(78.3 \%)$ and the $\mathrm{BSA} \leq 3 \%$ group $(70.6 \%)$ compared with the BSA $>3 \%$ group (44.8\%; Fig. $6 \mathrm{c}$ ).

\section{DISCUSSION}

This analysis demonstrated that deucravacitinib treatment produced early, clinically meaningful, and similar improvements across several assessments of clinical response (PASI, BSA, sPGA). These improvements were mirrored by QoL improvements, as measured by DLQI. At the three highest dosages tested, a substantial percentage of the deucravacitinib-treated patients met thresholds of clinically meaningful improvement in PASI (PASI 75, PASI 90, PASI $100)$ as well as treat-to-target BSA values recommended by the National Psoriasis
Foundation (BSA $\leq 1 \%$ at 12 weeks) $[6,18]$ and acceptable response (BSA $\leq 3 \%$ or BSA improvement $\geq 75 \%$ at 12 weeks of treatment).

At Week 12, the percentages of patients with PASI 75, PASI 90, PASI 100, and sPGA 0/1 were substantially higher in the deucravacitinib dosage groups than in the placebo group. In the main analysis of this Phase 2 trial, an sPGA score of $0 / 1$ was achieved in $7 \%$ of the placebo group and in $76 \%$ of the deucravacitinib $3 \mathrm{mg}$ BID group, $75 \%$ of the $12 \mathrm{mg}$ QD group, and $64 \%$ of the $6 \mathrm{mg}$ BID group at Week 12 [5]. Additionally, up to $38.6 \%$ and $56.8 \%$ of patients combined across the three deucravacitinib dosage groups achieved $\mathrm{BSA} \leq 1 \%$ and $\leq 3 \%$, respectively. As would be expected in this patient population, at Week 12 a similar proportion of patients in the combined deucravacitinib dosage group achieved sPGA $\times$ BSA 75 and PASI 75. Comparable findings from other studies that also used a composite assessment with BSA suggest $\mathrm{sPGA} \times \mathrm{BSA}$ may be a viable alternative to PASI that may be used in both clinical trials and clinical practice settings to assess severity of psoriasis [12, 19-21]. Treatment with deucravacitinib resulted in greater improvements in QoL (as assessed by DLQI) versus placebo, which

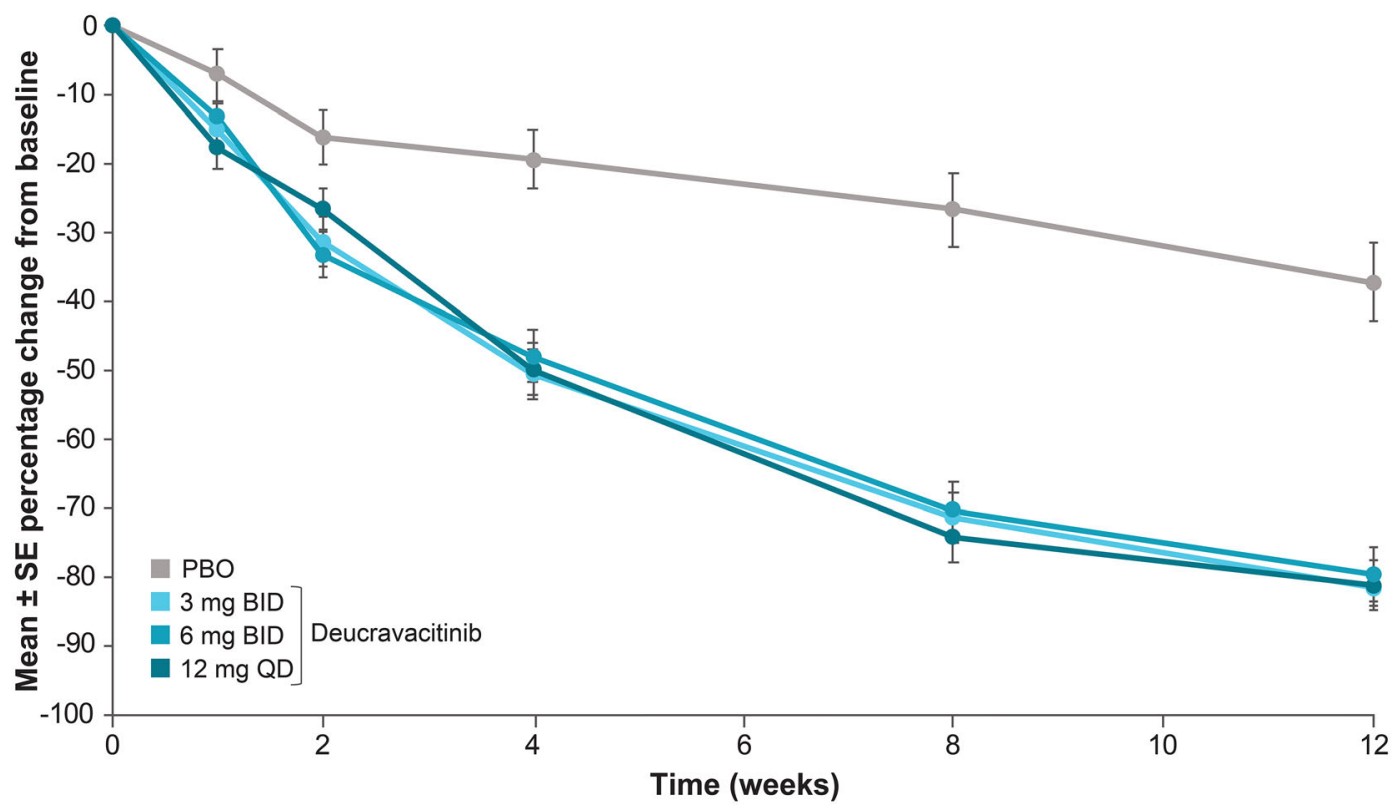

Fig. 1 Mean percentage change from baseline in PASI scores over time for placebo and the deucravacitinib $3 \mathrm{mg}$ BID, $6 \mathrm{mg}$ BID, and $12 \mathrm{mg}$ QD dosage groups. BID twice daily; PASI Psoriasis Area and Severity Index; PBO placebo; $Q D$ once daily 


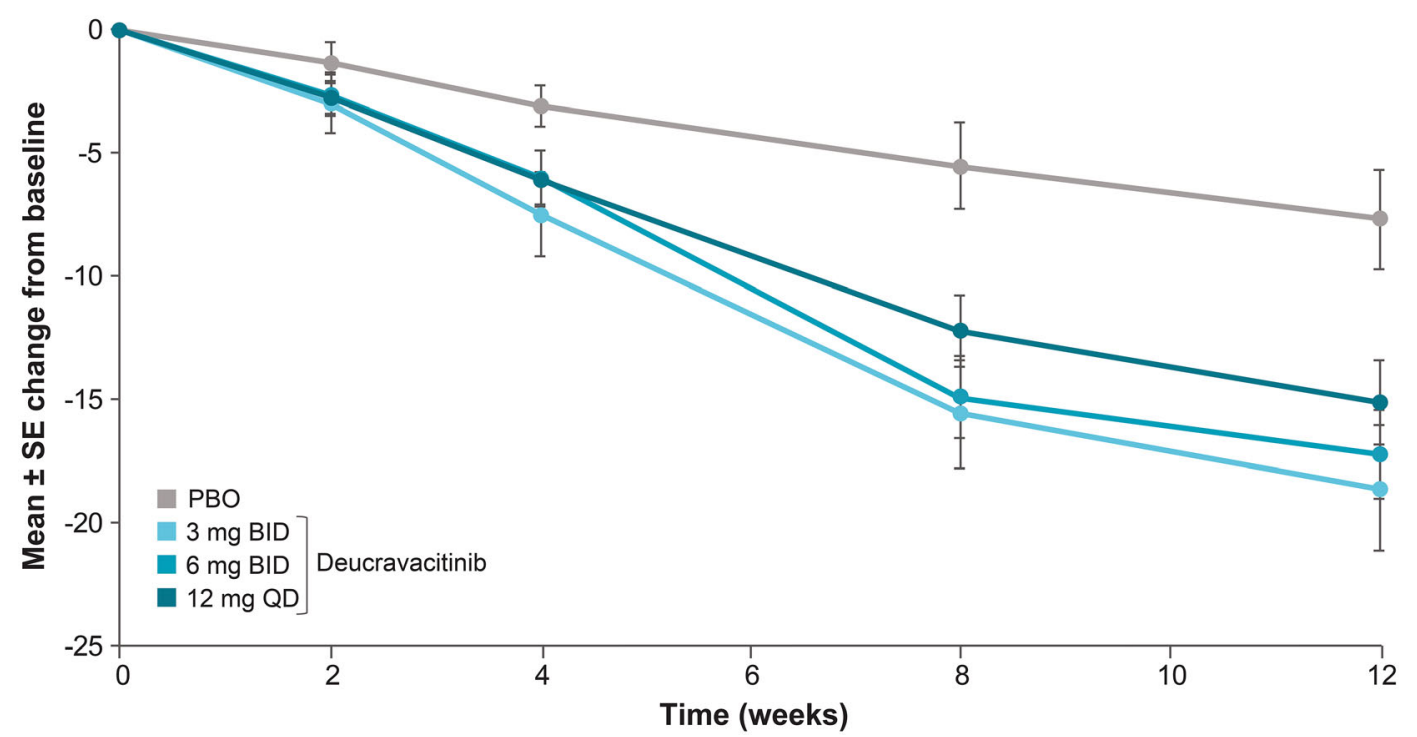

Fig. 2 Mean absolute change from baseline in BSA percentage over time for placebo and the deucravacitinib $3 \mathrm{mg}$ BID, $6 \mathrm{mg}$ BID, and $12 \mathrm{mg}$ QD dosage groups. $B I D$ twice daily; $B S A$ body surface area; $P B O$ placebo; $Q D$ once daily

occurred by Week 4. The improvements in QoL paralleled increases in the rates of PASI 75, PASI 90, PASI 100, and sPGA $0 / 1$ and were commensurate with improvements in absolute PASI and BSA scores over time through Week 12.

Consistency between clinical and QoL outcomes is not unexpected, given the association between disease severity and QoL and the volume of literature reporting a substantial negative impact of psoriasis on QoL and, conversely, greater improvement in QoL mirroring an increase in skin clearance [3, 9, 22-27]. Previous studies have demonstrated consistency between physician-reported clinical outcomes and patient-reported outcomes such as QoL $[25,28-33]$. In addition to the similar pattern of responses for clinical and QoL outcomes reported here, the current analysis also showed that a large percentage of patients achieved DLQI 0/1 despite absence of complete skin clearance (see Fig. 6) and despite having BSA involvement $>3 \%$ [6]. Awareness has increased over the last decade that patients with psoriasis experience effects on QoL beyond their level of skin involvement $[25,34]$. Furthermore, the association between objective clinical response and QoL may vary depending on BSA affected [23], QoL domain (e.g., mental vs. physical) [35], QoL assessment tool (e.g., the dermatology-specific measure DLQI vs generic health status measures) [26], ethnicity [36, 37], sex [38], culture [36], and socioeconomic status $[36,38]$. The current analysis and other studies in psoriasis highlight the importance of patientreported outcomes, in addition to clinician-reported outcomes and other traditional clinical severity tools, when assessing treatment efficacy and the impact of psoriasis on daily life with effective agents [6, 25-27, 31, 39-43].

The limitations of this post hoc analysis must be considered. Differences between deucravacitinib and placebo in clinical and patientreported outcomes were not multiplicity-adjusted for statistical significance. Analysis of the rate of achievement of DLQI $0 / 1$ by PASI response bands was limited by the relatively small number of patients in individual PASI categories [5]. It should also be noted that all of the instruments available for assessing clinical outcomes or QoL have limited precision and truncation associated with the limitations of data capture. Finally, the relatively short 12-week treatment period and small sample size in the Phase 2 trial presented another limitation for this analysis. Larger, longer-term Phase 3 trials of deucravacitinib in psoriasis are ongoing or have been completed (POETYK PSO-1 [NCT03624127] [44]; POETYK PSO-2 


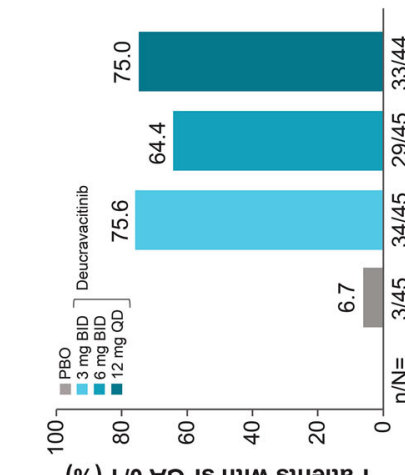

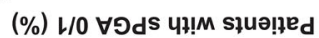
o

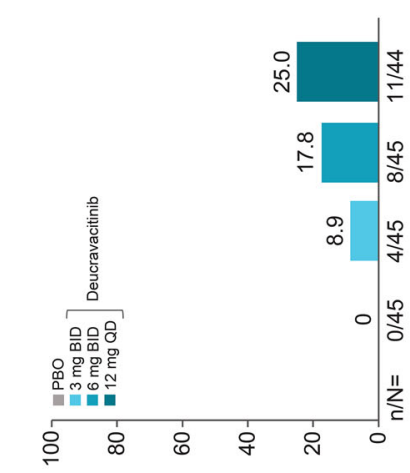
○

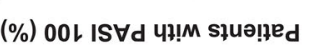

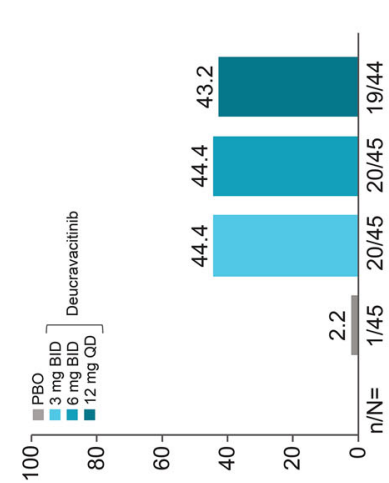

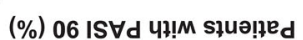

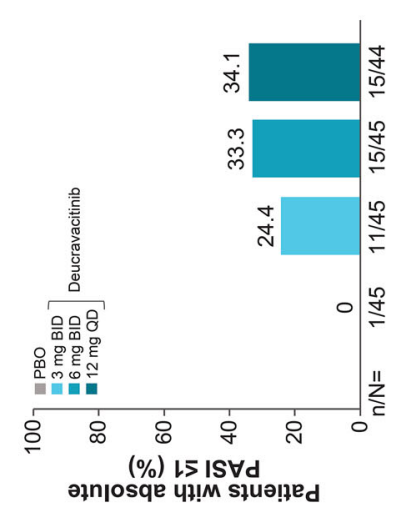

4

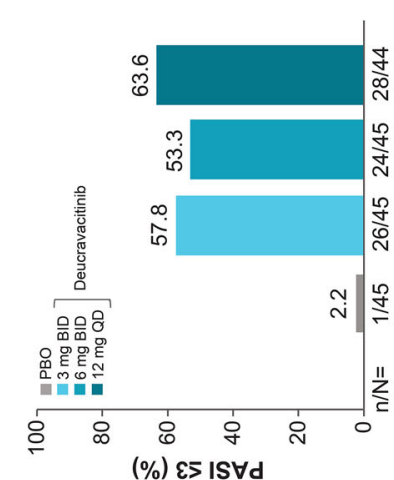

0

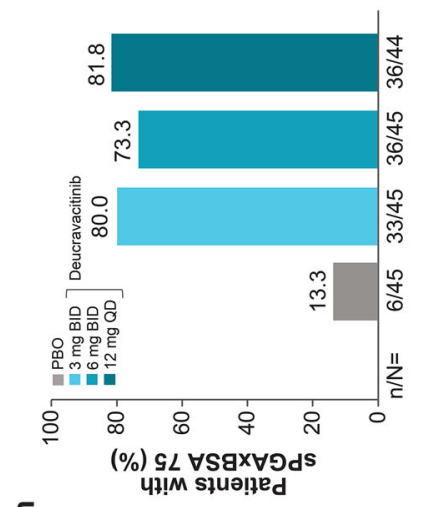

上
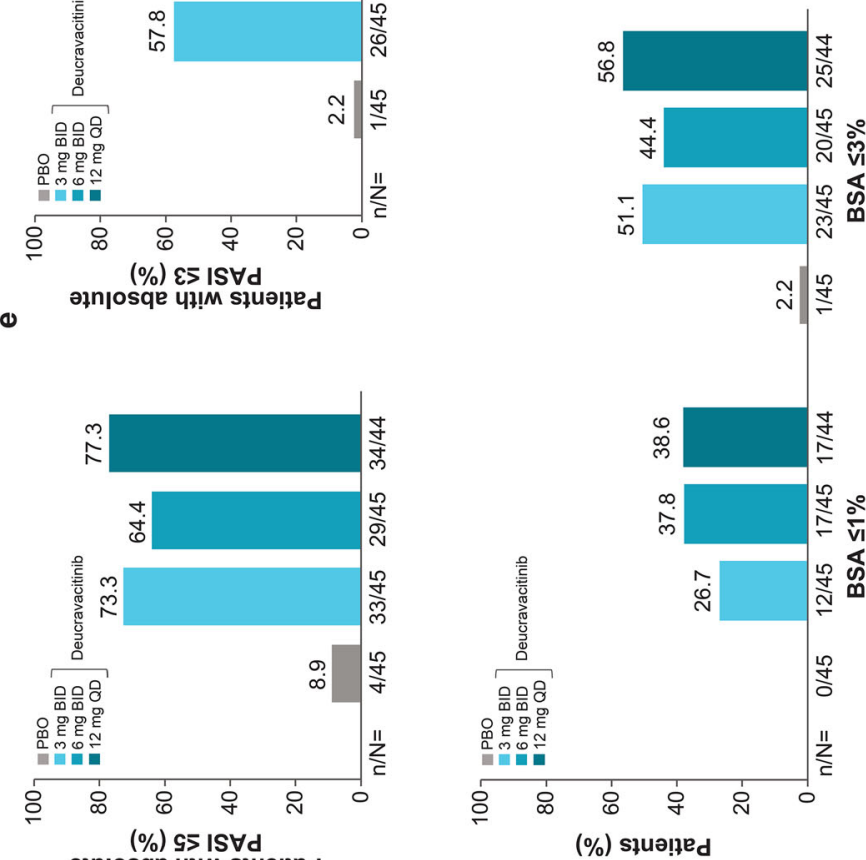

월

VI

$\varangle \frac{0}{0}$

010 ส

का से

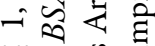

VI $\cong$.

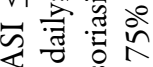

$\simeq$ \&

记

윰

$\approx \exists n$

$+\dot{0} \Xi$

ஸे 을

VI 애용 $x$

ज ธิธ

$\leq$ 过

च

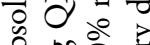
ते

ヘ

VI $\stackrel{0}{\square}$

幽

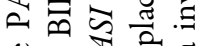

艺

○ 0 这

ति 0 茷

$\tau$

in 00.

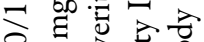

$m$ व

$\varangle$ 음

क $\Xi \backsim$

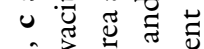

$8 \overbrace{}^{2}$ 它

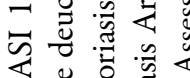

a $\subseteq$ 焉

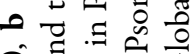

尺े जी

๑ ㅋ.ّ

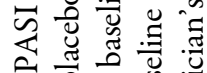

$\rightarrow$ ह

के 0ีث

ำ

저ㄴㅠㅠ

चै صิ

$\times \times$

ㄴ

UN

矛部 8

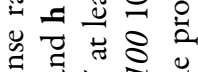

ํำ至

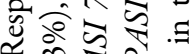

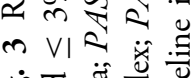

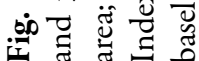




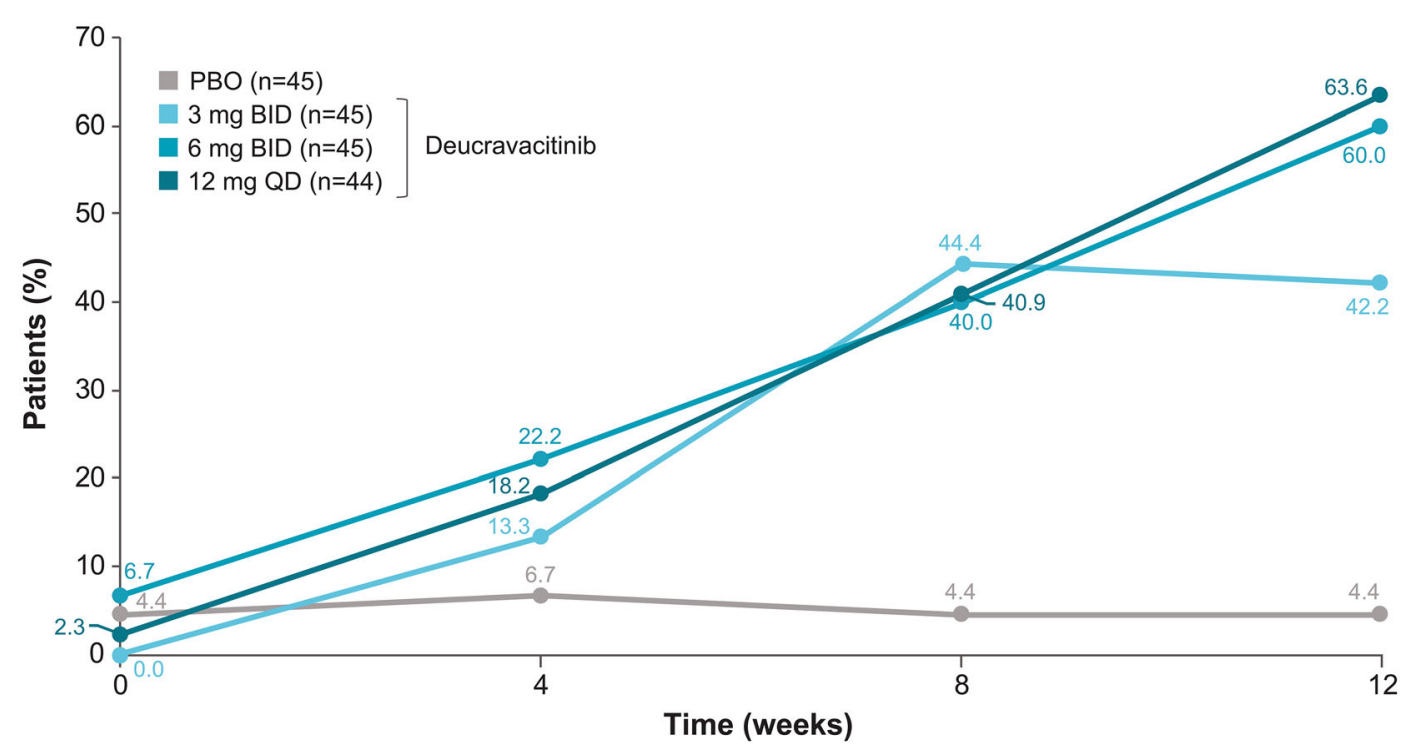

Fig. 4 Percentage of patients with a DLQI overall score of $0 / 1$, which reflects no impact on patient's life, for placebo and the deucravacitinib $3 \mathrm{mg}$ BID, $6 \mathrm{mg}$ BID, and $12 \mathrm{mg}$
QD dosage groups. BID twice daily; DLQI Dermatology Life Quality Index; $Q D$ every day; $Q D L$ quality of life

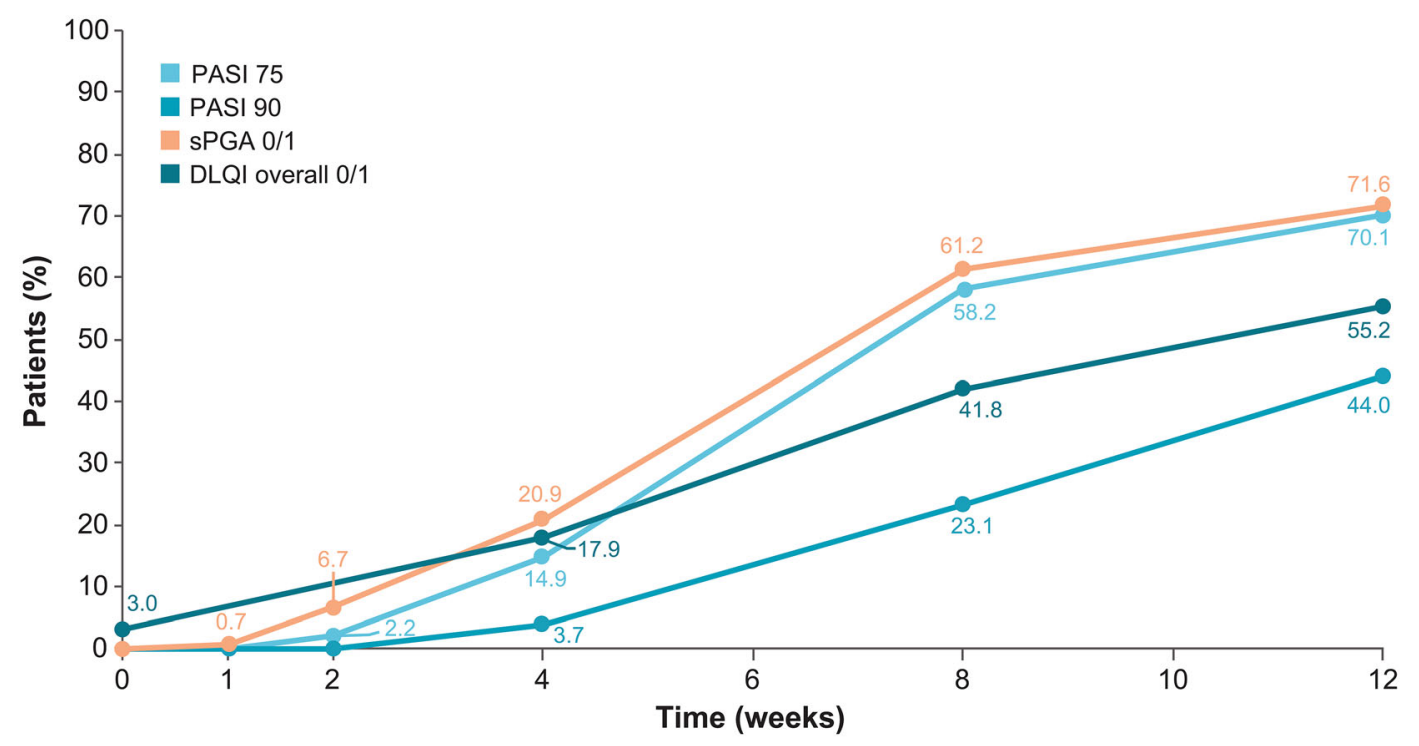

Fig. 5 Time course of PASI 75, PASI 90, sPGA 0/1, and DLQI overall score of $0 / 1$ response rates for the combined deucravacitinib $3 \mathrm{mg}$ BID, $6 \mathrm{mg} \mathrm{BID}$, and $12 \mathrm{mg}$ QD dosage groups. BID twice daily; DLQI Dermatology Life Quality Index; PASI 75 at least $75 \%$ reduction from

[NCT03611751];

[NCT04167462];

[NCT03924427];

[NCT04036435]) and will provide important baseline in Psoriasis Area and Severity Index; PASI 90 at least $90 \%$ reduction from baseline in Psoriasis Area and Severity Index; $Q D$ every day; $s P G A$ static Physician's Global Assessment

data regarding consistency between clinical outcomes and QoL in a larger population of psoriasis patients over longer follow-up periods. 
a

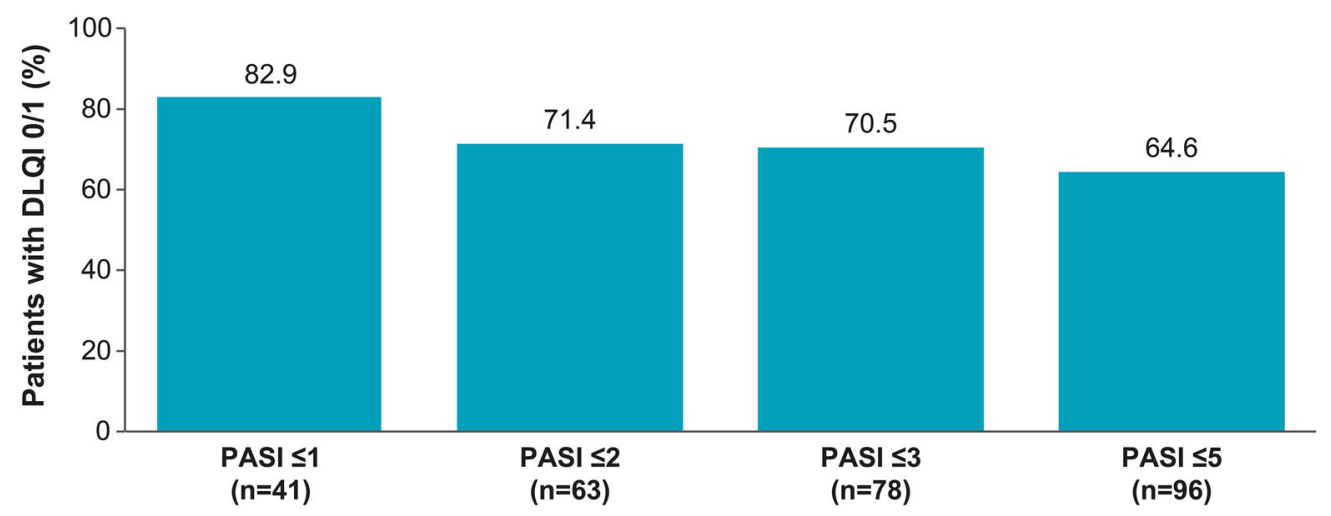

b

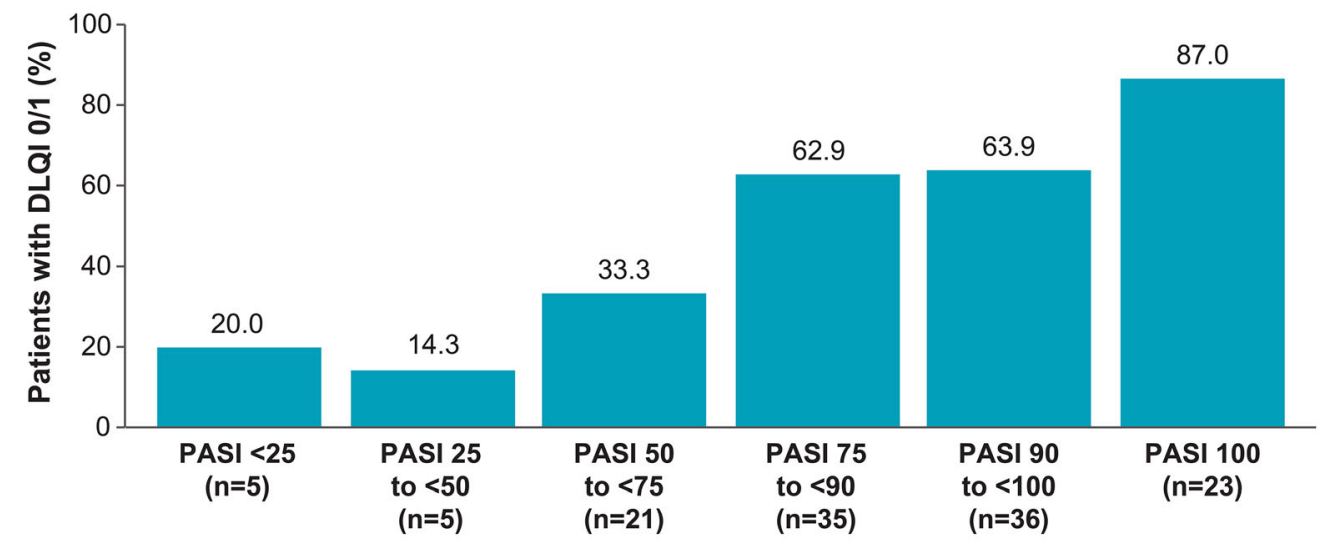

C

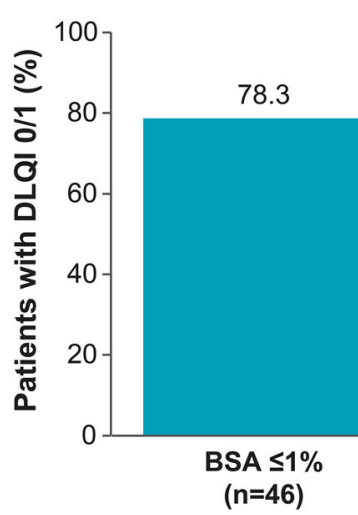

Fig. 6 DLQI $0 / 1$ at Week 12 by absolute PASI (a), PASI response band (b), and BSA response band (c) for the combined patient groups of deucravacitinib $3 \mathrm{mg}$ BID, $6 \mathrm{mg} \mathrm{BID}$, and $12 \mathrm{mg}$ QD dosage groups. BID twice daily; $B S A$ body surface area; DLQI Dermatology Life Quality Index; PASI Psoriasis Area and Severity Index; PASI < $25<25 \%$ improvement from baseline Psoriasis Area and
70.6



Severity Index; PASI 25 to $<5025 \%$ to $<50 \%$ improvement; PASI 50 to $<7550 \%$ to $<75 \%$ improvement; PASI 75 to $<9075 \%$ to $<90 \%$ improvement; PASI 90 to $<10090 \%$ to $<100 \%$ improvement; PASI $100100 \%$ improvement from baseline Psoriasis Area and Severity Index; $Q D$ once daily 


\section{CONCLUSION}

This post hoc analysis of the Phase 2 trial demonstrated that deucravacitinib at doses of $\geq 3 \mathrm{mg}$ BID provided early improvements across multiple assessments of clinical efficacy as well as QoL in patients with moderate to severe plaque psoriasis, suggesting that deucravacitinib may be a promising therapy for this population. Early concurrent trends in improvement occurred over the 12-week trial across different assessments, with no impact on life (DLQI 0/1) observed in many patients who did not achieve complete skin clearance.

\section{ACKNOWLEDGEMENTS}

The authors thank the patients for their participation, as well as the investigators and their teams, and biostatisticians for their contributions.

Funding. This analysis was sponsored by Bristol Myers Squibb. Bristol Myers Squibb also funded the journal's Rapid Service Fees.

Medical writing assistance. Professional medical writing from Barbara Zeman, PhD, and editorial assistance were provided by Peloton Advantage, LLC, an OPEN Health company, Parsippany, NJ, and were funded by Bristol Myers Squibb.

Authorship. All named authors meet the International Committee of Medical Journal Editors (ICMJE) criteria for authorship for this article, take responsibility for the integrity of the work as a whole, and have given their approval for this version to be published.

Author contributions. DT, BS, LP, MAM, MJC, RMK, AAN, SB, JFM, and ABG contributed to the conception and study design and data interpretation. $\mathrm{KBG}, \mathrm{PF}, \mathrm{MG}, \mathrm{AM}$, and $\mathrm{KAP}$ contributed to data acquisition and data interpretation. YE and JY contributed to data analysis. LW contributed to conception or design or data analysis. All authors reviewed the manuscript and gave final approval for publication.

Disclosures. Diamant Thaçi has received honoraria and lecture fees from AbbVie, Almirall, Amgen, Biogen Idec, Boehringer Ingelheim, Bristol Myers Squibb, Janssen, Leo Pharma, Novartis, Pfizer, Roche-Posay, Sandoz-Hexal, Sanofi, Target-Solution, and UCB. Bruce Strober has received honoraria from AbbVie, Almirall, Amgen, Arcutis, Arena, Aristea, Boehringer Ingelheim, Bristol Myers Squibb, Connect Biopharma, Dermavant, Eli Lilly, Equillium, GlaxoSmithKline, Immunic Therapeutics, Janssen, Leo Pharma, Maruho, Meiji Seika Pharma, Mindera, Novartis, Ortho Dermatologics, Pfizer, Regeneron, Sanofi Genzyme, Sun Pharma, and UCB; has served as an investigator for AbbVie, Cara, CorEvitas' (Corrona) Psoriasis Registry, Dermavant, Dermira, and Novartis; has served as a scientific director (consultant fee) for CorEvitas' (Corrona) Psoriasis Registry; and has served as a speaker for AbbVie, Amgen, Eli Lilly, Janssen, and Sanofi Genzyme. Kenneth B. Gordon has served as a consultant for AbbVie, Amgen, Almirall, Arcutis, Arena Pharma, Boehringer Ingelheim, Bristol Myers Squibb, Celgene, Dermavant, Dermira, Eli Lilly, Incyte, Janssen, Kyowa Hakko Kirin, Leo Pharma, Novartis, Pfizer, Sanofi Genzyme, Sun Pharma, and $\mathrm{UCB}$, and has received research grants from AbbVie, Boehringer Ingelheim, Bristol Myers Squibb, Celgene, Eli Lilly, Janssen, Novartis, and UCB. Peter Foley has received grant support from AbbVie, Amgen, Celgene, Eli Lilly, Janssen, Leo Pharma, Merck, Novartis, Pfizer, Sanofi, and Sun Pharma; has served as an investigator for AbbVie, Amgen, AstraZeneca, Arcutis, Aslan, Bristol Myers Squibb, Boehringer Ingelheim, Botanix, Celgene, Celtaxsys, CSL, Cutanea, Dermira, Eli Lilly, Galderma, Genentech, GlaxoSmithKline, Hexima, Janssen, Leo Pharma, Merck, Novartis, Pfizer, Regeneron, Reistone, Roche, Sanofi, Sun Pharma, UCB, and Valeant; has served as an advisory board member for AbbVie, Amgen, Bristol Myers Squibb, Boehringer Ingelheim, Celgene, Eli Lilly, Galderma, GlaxoSmithKline, Janssen, Leo Pharma, Merck, Novartis, Pfizer, Sanofi, Sun Pharma, UCB, and Valeant; has served as a consultant for 
Bristol Myers Squibb, Eli Lilly, Galderma, Janssen, Leo Pharma, Novartis, Pfizer, Roche, and UCB; has received travel grants from AbbVie, Eli Lilly, Galderma, Janssen, Leo Pharma, Merck, Novartis, Pfizer, Roche, Sun Pharma, and Sanofi; and has served as a speaker for or received honoraria from AbbVie, Celgene, Eli Lilly, Galderma, GlaxoSmithKline, Janssen, Leo Pharma, Merck, Novartis, Pfizer, Roche, and Valeant. Melinda Gooderham has served as an advisory board member for AbbVie, Amgen, Arcutis, Bausch Health, Boehringer Ingelheim, Bristol Myers Squibb, Eli Lilly, Galderma, Janssen, Leo Pharma, Novartis, Pfizer, Regeneron, Sanofi Genzyme, Sun Pharma, and UCB; has served as a consultant for AbbVie, Akros, Amgen, Arcutis, Boehringer Ingelheim, Eli Lilly, Galderma, Janssen, Kyowa Kirin, Leo Pharma, Novartis, Pfizer, Sanofi Genzyme, and UCB; has served as an investigator for AbbVie, Akros, Amgen, Arcutis, Bausch Health, Boehringer Ingelheim, Bristol Myers Squibb, Celgene, Dermira, Dermavant, Eli Lilly, Galderma, GlaxoSmithKline, Incyte, Janssen, Kyowa Kirin, Leo Pharma, Merck, MedImmune, Novartis, Pfizer, Regeneron, Roche, Sanofi Genzyme, Sun Pharma, Takeda, and UCB; and has served on a speakers bureau for AbbVie, Amgen, Arcutis, Boehringer Ingelheim, Bristol Myers Squibb, Celgene, Eli Lilly, Galderma, Janssen, Leo Pharma, Novartis, Pfizer, Regeneron, Roche, Sanofi Genzyme, Sun Pharma, and UCB. Akimichi Morita has received honoraria from AbbVie GK, Ayumi Pharmaceutical, Boehringer Ingelheim Japan, Celgene K.K., Eisai, Eli Lilly Japan K.K., Inforward, Janssen Pharmaceutical K.K., Kyowa Kirin, Maruho, Mitsubishi Tanabe Pharma, Nippon Kayaku, Novartis Pharma K.K., Taiho Pharmaceutical, Torii Pharmaceutical, and Ushio; has received research support for AbbVie GK, Eisai, Eli Lilly Japan K.K., Kyowa Hakko Kirin, Leo Pharma KK, Maruho, Mitsubishi Tanabe Pharma, Novartis Pharma K.K., Taiho Pharmaceutical, and Torii Pharmaceutical; and has served as a consultant for AbbVie GK, Boehringer Ingelheim Japan, Bristol Myers Squibb, Celgene K.K., Eli Lilly Japan K.K., GlaxoSmithKline K.K., Janssen Pharmaceutical K.K., Kyowa Kirin, Maruho, Mitsubishi Tanabe Pharma, Nichi-Iko Pharmaceutical, Nippon
Kayaku, Novartis Pharma K.K., NPO Health Institute Research of Skin, Pfizer Japan, Sun Pharmaceutical Industries, Torii Pharmaceutical, and UCB Japan. Kim A. Papp has served as a consultant for AbbVie, Akros, Amgen, Arcutis, Astellas, AstraZeneca, Baxalta, Baxter, Boehringer Ingelheim, Bristol Myers Squibb, CanFite, Celgene, Coherus, Dermira, Dow Pharma, Eli Lilly, Forward Pharma, Galderma, Genentech, Janssen, Kyowa Hakko Kirin, Leo Pharma, Meiji Seika Pharma, Merck Sharp \& Dohme, Merck Serono, Mitsubishi Pharma, Novartis, Pfizer, Regeneron, Roche, Sanofi Genzyme, Takeda, UCB, and Valent; has received honoraria from AbbVie, Akros, Amgen, Baxter, Boehringer Ingelheim, Celgene, Coherus, Eli Lilly, Forward Pharma, Galderma, GlaxoSmithKline, Janssen, Kyowa Hakko Kirin, Merck Sharp \& Dohme, Merck Serono, Novartis, Pfizer, Takeda, UCB, and Valeant; has received research grants from AbbVie, Akros, Allergan, Amgen, Anacor, Arcutis, AstraZeneca, Baxalta, Boehringer Ingelheim, Bristol Myers Squibb, Celgene, Coherus, Dermira, Dow Pharma, Eli Lilly, Galderma, Genentech, GlaxoSmithKline, Janssen, Kyowa Hakko Kirin, Leo Pharma, MedImmune, Meiji Seika Pharma, Merck Serono, Novartis, Pfizer, Regeneron, Roche, Sanofi-Aventis/Genzyme, Takeda, UCB, and Valeant; has served on a scientific officer/steering committee/advisory board for AbbVie, Akros, Amgen, Anacor, Astellas, Baxter, Boehringer Ingelheim, Bristol Myers Squibb, Celgene, Dow Pharma, Eli Lilly, Galderma, Janssen, Kyowa Hakko Kirin, Merck Sharp \& Dohme, Merck Serono, Novartis, Pfizer, Regeneron, Sanofi Genzyme, and Valeant; and has served on the speakers bureau for AbbVie, Amgen, Astellas, Celgene, Eli Lilly, Galderma, Janssen, Kyowa Hakko Kirin, Leo Pharma, Merck Sharp \& Dohme, Novartis, Pfizer, and Valeant. Lluís Puig has received honoraria or consultation fees from AbbVie, Almirall, Amgen, Baxalta, Biogen, Boehringer Ingelheim, Celgene, Eli Lilly, Fresenius Kabi, Gebro, Janssen, Leo Pharma, Merck-Serono, Merck Sharp \& Dohme, Mylan, Novartis, Pfizer, Regeneron, Roche, Samsung Bioepis, Sandoz, Sanofi, and $\mathrm{UCB}$; has received research grants from or participated in clinical trials (paid to institution) for AbbVie, Almirall, Amgen, Boehringer 
Ingelheim, Celgene, Eli Lilly, Janssen, Leo Pharma, Novartis, Pfizer, Regeneron, Roche, Sanofi, and UCB; and has served on the speakers bureau for Celgene, Eli Lilly, Janssen, Merck Sharp \& Dohme, Novartis, and Pfizer. M. Alan Menter has served on the advisory board for Abbott Labs, Amgen, Boehringer Ingelheim, Janssen Biotech, and Leo Pharma; has served as a consultant for AbbVie, Amgen, Eli Lilly, Janssen Biotech, Leo Pharma, Novartis, Sun Pharma, and UCB; has received honoraria from AbbVie, Amgen, Boehringer Ingelheim, Eli Lilly, Janssen Biotech, Leo Pharma, Novartis, Sun Pharma, and UCB; has served as an investigator for AbbVie, Amgen, Boehringer Ingelheim, Celgene, Eli Lilly, Janssen Biotech, Leo Pharma, Merck, Novartis, Sun Pharma, and UCB; has received research grants from AbbVie, Amgen, Boehringer Ingelheim, Celgene, Janssen Biotech, Leo Pharma, Merck, and Sun Pharma; and has served as a speaker for AbbVie, Amgen, Janssen Biotech, Leo Pharma, Sun Pharma, and UCB. Matthew J. Colombo, Yedid Elbez, Renata M. Kisa, June Ye, Andrew A. Napoli, Lan Wei, and Subhashis Banerjee are employees of and shareholders in Bristol Myers Squibb. Joseph F. Merola has served as a consultant and/or investigator for AbbVie, Amgen, Biogen, Bristol Myers Squibb, Dermavant, Eli Lilly, Janssen, Leo Pharma, Novartis, Pfizer, Regeneron, Sanofi, Sun Pharma, and UCB. Alice B. Gottlieb has received consultation fees from AnaptysBio, Avotres, Boehringer Ingelheim, Incyte, Lilly ICOS, Novartis, Pfizer, and Sun Pharma; has served on an advisory board for Amgen, Bristol Myers Squibb, Celgene, Janssen, Janssen-Ortho, Leo Pharma, Novartis, and UCB; and has served as an investigator for Janssen-Ortho, Boehringer Ingelheim, Incyte, Novartis, Sun Pharma, UCB, and XBiotech.

Compliance with ethics guidelines. The trial was conducted in accordance with Good Clinical Practice guidelines defined by the International Council for Harmonisation, the Declaration of Helsinki, and the laws and regulatory requirements of the countries with participating study and the institutional review boards or independent ethics committees at each site (see Supporting Information). All patients provided written informed consent prior to any study procedures in accordance with Good Clinical Practice and local legislation.

Data availability. The Bristol Myers Squibb policy on data sharing may be found at https:// www.bms.com/researchers-and-partners/ independent-research/data-sharing-requestprocess.html.

Open Access. This article is licensed under a Creative Commons Attribution-NonCommercial 4.0 International License, which permits any non-commercial use, sharing, adaptation, distribution and reproduction in any medium or format, as long as you give appropriate credit to the original author(s) and the source, provide a link to the Creative Commons licence, and indicate if changes were made. The images or other third party material in this article are included in the article's Creative Commons licence, unless indicated otherwise in a credit line to the material. If material is not included in the article's Creative Commons licence and your intended use is not permitted by statutory regulation or exceeds the permitted use, you will need to obtain permission directly from the copyright holder. To view a copy of this licence, visit http://creativecommons.org/licenses/by$\mathrm{nc} / 4.0 /$.

\section{REFERENCES}

1. Spuls PI, Lecluse LL, Poulsen ML, Bos JD, Stern RS, Nijsten T. How good are clinical severity and outcome measures for psoriasis? Quantitative evaluation in a systematic review. J Invest Dermatol. 2010;130(4):933-43.

2. Feldman SR, Krueger GG. Psoriasis assessment tools in clinical trials. Ann Rheum Dis. 2005;64(Suppl 2): ii65-8 (discussion ii9-73).

3. Menter A, Gottlieb A, Feldman SR, Van Voorhees AS, Leonardi CL, Gordon KB, et al. Guidelines of care for the management of psoriasis and psoriatic arthritis: Section 1. Overview of psoriasis and guidelines of care for the treatment of psoriasis with biologics. J Am Acad Dermatol. 2008;58(5):826-50. 
4. Fredriksson T, Pettersson U. Severe psoriasis-oral therapy with a new retinoid. Dermatologica. 1978;157(4):238-44.

5. Papp K, Gordon K, Thaçi D, Morita A, Gooderham $\mathrm{M}$, Foley $\mathrm{P}$, et al. Phase 2 trial of selective tyrosine kinase 2 inhibition in psoriasis. $\mathrm{N}$ Engl J Med. 2018;379(14):1313-21.

6. Armstrong AW, Siegel MP, Bagel J, Boh EE, Buell M, Cooper KD, et al. From the Medical Board of the National Psoriasis Foundation: treatment targets for plaque psoriasis. J Am Acad Dermatol. 2017;76(2): 290-8.

7. Weisman S, Pollack CR, Gottschalk RW. Psoriasis disease severity measures: comparing efficacy of treatments for severe psoriasis. J Dermatol Treat. 2003;14(3):158-65.

8. Finlay AY, Khan GK. Dermatology Life Quality Index (DLQI)-a simple practical measure for routine clinical use. Clin Exp Dermatol. 1994;19(3): 210-6.

9. Katugampola RP, Lewis VJ, Finlay AY. The Dermatology Life Quality Index: assessing the efficacy of biological therapies for psoriasis. Br J Dermatol. 2007;156(5):945-50.

10. Nogueira M, Puig L, Torres T. JAK inhibitors for treatment of psoriasis: focus on selective TYK2 inhibitors. Drugs. 2020;80(4):341-51.

11. Burke JR, Cheng L, Gillooly KM, Strnad J, ZupaFernandez A, Catlett IM, et al. Autoimmune pathways in mice and humans are blocked by pharmacological stabilization of the TYK2 pseudokinase domain. Sci Transl Med. 2019;11(502):1-16.

12. Walsh JA, McFadden M, Woodcock J, Clegg DO, Helliwell P, Dommasch E, et al. Product of the Physician Global Assessment and body surface area: a simple static measure of psoriasis severity in a longitudinal cohort. J Am Acad Dermatol. 2013;69(6):931-7.

13. Chow C, Simpson MJ, Luger TA, Chubb H, Ellis CN. Comparison of three methods for measuring psoriasis severity in clinical studies (Part 1 of 2): change during therapy in Psoriasis Area and Severity Index, Static Physician's Global Assessment and Lattice System Physician's Global Assessment. J Eur Acad Dermatol Venereol. 2015;29(7):1406-14.

14. Simpson MJ, Chow C, Morgenstern H, Luger TA, Ellis CN. Comparison of three methods for measuring psoriasis severity in clinical studies (part 2 of 2): use of quality of life to assess construct validity of the Lattice System Physician's Global Assessment, Psoriasis Area and Severity Index and static
Physician's Global Assessment. J Eur Acad Dermatol Venereol. 2015;29(7):1415-20.

15. Stein Gold L, Hansen JB, Patel D, Veverka KA, Strober B. PGAxBSA composite versus PASI: comparison across disease severities and as therapeutic response measure for $\mathrm{Cal} / \mathrm{BD}$ foam in plaque psoriasis. J Am Acad Dermatol. 2020;83(1):131-8.

16. Strober B, Gottlieb AB, Thaçi D, Puig L, Colombo MJ, Kundu S, et al. An oral, selective tyrosine kinase 2 inhibitor, deucravacitinib (BMS-986165), reduced absolute psoriasis area and severity index in a phase 2 trial in psoriasis [poster]. Presented at: the Spring Symposium of the European Academy of Dermatology and Venereology; 2020.

17. Hongbo Y, Thomas CL, Harrison MA, Salek MS, Finlay AY. Translating the science of quality of life into practice: what do dermatology life quality index scores mean? J Invest Dermatol. 2005;125(4): 659-64.

18. Treat to target: National Psoriasis Foundation, 2021. https://www.psoriasis.org/treat-to-target/\#: $\sim:$ text=The $\% 20$ idea $\% 20$ behind $\% 20$ the $\%$

20treatment,of\%20your\%20body\%20surface\% 20area. Accessed 13 Jul 2021.

19. Gottlieb AB, Merola JF, Chen R, Levi E, Duffin KC. Assessing clinical response and defining minimal disease activity in plaque psoriasis with the Physician Global Assessment and body surface area (PGA $\times$ BSA) composite tool: an analysis of apremilast phase 3 ESTEEM data. J Am Acad Dermatol. 2017;77(6):1178-80.

20. Gottlieb AB, Germino R, Herrera V, Meng X, Merola JF. Exploration of the product of the 5-point Investigator's Global Assessment and Body Surface Area $($ IGA $\times$ BSA) as a practical minimal disease activity goal in patients with moderate-to-severe psoriasis. Dermatology. 2019;235(4):348-54.

21. Merola JF, Amato DA, See K, Burge R, Mallinckrodt $\mathrm{C}$, Ojeh CK, et al. Evaluation of sPGA $\times$ BSA as an outcome measure and treatment target for clinical practice. J Invest Dermatol. 2018;138(9):1955-61.

22. Armstrong AW, Schupp C, Wu J, Bebo B. Quality of life and work productivity impairment among psoriasis patients: findings from the National Psoriasis Foundation survey data 2003-2011. PLoS ONE. 2012;7(12):e52935.

23. Armstrong AW, Villanueva Quintero DG, Echeverría CM, Gu Y, Karunaratne M, Reyes SO. Body region involvement and quality of life in psoriasis: analysis of a randomized controlled trial of adalimumab. Am J Clin Dermatol. 2016;17(6):691-9. 
24. Hrehorow E, Salomon J, Matusiak L, Reich A, Szepietowski JC. Patients with psoriasis feel stigmatized. Acta Derm Venereol. 2012;92(1):67-72.

25. Lacour JP, Bewley A, Hammond E, Hansen JB, Horne L, Paul C, et al. Association between patientand physician-reported outcomes in patients with moderate-to-severe plaque psoriasis treated with biologics in real life (PSO-BIO-REAL). Dermatol Ther. 2020;10(5):1099-109.

26. Revicki DA, Willian MK, Menter A, Saurat JH, Harnam N, Kaul M. Relationship between clinical response to therapy and health-related quality of life outcomes in patients with moderate to severe plaque psoriasis. Dermatology. 2008;216(3):260-70.

27. Dubertret L, Mrowietz U, Ranki A, van de Kerkhof PC, Chimenti S, Lotti T, et al. European patient perspectives on the impact of psoriasis: the EUROPSO patient membership survey. Br J Dermatol. 2006;155(4):729-36.

28. Puig L, Dossenbach M, Berggren L, Ljungberg A, Zachariae C. Absolute and relative psoriasis area and severity indices (PASI) for comparison of the efficacy of ixekizumab to etanercept and placebo in patients with moderate-to-severe plaque psoriasis: an integrated analysis of UNCOVER-2 and UNCOVER-3 outcomes. Acta Derm Venereol. 2019;99(11):971-7.

29. Papp KA, Barber K, Bissonnette R, Bourcier M, Lynde CW, Poulin Y, et al. Improvements in patient-reported outcomes in patients with psoriasis receiving etanercept plus topical therapies: results from REFINE. J Eur Acad Dermatol Venereol. $2015 ; 29(8): 1555-61$.

30. Takeshita J, Callis Duffin K, Shin DB, Krueger GG, Robertson AD, Troxel AB, et al. Patient-reported outcomes for psoriasis patients with clear versus almost clear skin in the clinical setting. J Am Acad Dermatol. 2014;71(4):633-41.

31. Gordon KB, Kimball AB, Chau D, Viswanathan HN, Li J, Revicki DA, et al. Impact of brodalumab treatment on psoriasis symptoms and health-related quality of life: use of a novel patient-reported outcome measure, the Psoriasis Symptom Inventory. $\mathrm{Br}$ J Dermatol. 2014;170(3):705-15.

32. Leonardi C, Reich K, Foley P, Torii H, Gerdes S, Guenther L, et al. Efficacy and safety of ixekizumab through 5 years in moderate-to-severe psoriasis: long-term results from the UNCOVER-1 and UNCOVER-2 phase-3 randomized controlled trials. Dermatol Ther. 2020;10(3):431-47.

33. Nakamura M, Lee K, Jeon C, Sekhon S, Afifi L, Yan $\mathrm{D}$, et al. Guselkumab for the treatment of psoriasis:
A review of phase III trials. Dermatol Ther. 2017;7(3):281-92.

34. Poulin Y, Sheth P, Gu Y, Teixeira HD. Health-related quality of life worsens disproportionately to objective signs of psoriasis after withdrawal of adalimumab therapy. Dermatol Ther. 2014;4(1): 33-42.

35. Strand V, Sharp V, Koenig AS, Park G, Shi Y, Wang $B$, et al. Comparison of health-related quality of life in rheumatoid arthritis, psoriatic arthritis and psoriasis and effects of etanercept treatment. Ann Rheum Dis. 2012;71(7):1143-50.

36. Kwan Z, Bong YB, Tan LL, Lim SX, Yong AS, Ch'ng $\mathrm{CC}$, et al. Socioeconomic and sociocultural determinants of psychological distress and quality of life among patients with psoriasis in a selected multiethnic Malaysian population. Psychol Health Med. 2017;22(2):184-95.

37. Kaufman BP, Alexis AF. Psoriasis in skin of color: insights into the epidemiology, clinical presentation, genetics, quality-of-life impact, and treatment of psoriasis in non-white racial/ethnic groups. Am J Clin Dermatol. 2018;19(3):405-23.

38. Jung S, Lee SM, Suh D, Shin HT, Suh DC. The association of socioeconomic and clinical characteristics with health-related quality of life in patients with psoriasis: a cross-sectional study. Health Qual Life Outcomes. 2018;16(1):180.

39. Martin G, Young M, Aldredge L. Recommendations for initiating systemic therapy in patients with psoriasis. J Clin Aesthet Dermatol. 2019;12(4): $13-26$.

40. Greb JE, Goldminz AM, Elder JT, Lebwohl MG, Gladman DD, Wu JJ, et al. Psoriasis. Nat Rev Dis Primers. 2016;2:16082.

41. Helmick CG, Lee-Han H, Hirsch SC, Baird TL, Bartlett CL. Prevalence of psoriasis among adults in the US: 2003-2006 and 2009-2010 National Health and Nutrition Examination Surveys. Am J Prev Med. 2014;47(1):37-45.

42. Langley RG, Krueger GG, Griffiths CE. Psoriasis: epidemiology, clinical features, and quality of life. Ann Rheum Dis. 2005;64(Suppl 2):ii18-23 (discussion ii4-5).

43. Baker EL, Coleman CI, Reinhart KM, Phung OJ, Kugelman L, Chen W, et al. Effect of biologic agents on non-PASI outcomes in moderate-to-severe plaque psoriasis: systematic review and meta-analyses. Dermatol Ther. 2012;2(1):9.

44. Armstrong A, Gooderham M, Warren R, Papp K, Strober B, Thaçi D, et al. Efficacy and safety of 
deucravacitinib, an oral, selective tyrosine kinase 2 (TYK2) inhibitor, compared with placebo and apremilast in moderate to severe plaque psoriasis: results from the POETYK PSO-1 study [abstract]. Presented at: the Annual Meeting of the American Academy of Dermatology; 2021. 